\title{
IN SILICO STUDY OF SIRT1 ACTIVATORS USING A MOLECULAR DYNAMIC APPROACH
}

\author{
AZMINAH AZMINAH ${ }^{1,4}$, MAKSUM RADJI' ${ }^{2}$, ABDUL MUN'IM ${ }^{3}$, REZI RIADHI SYAHDI ${ }^{1}$, ARRY YANUAR ${ }^{1 *}$
}

${ }^{1}$ Laboratory of Biomedical Computation and Drug Design, Faculty of Pharmacy, Universitas Indonesia, Depok, West Java 16424, Indonesia. ${ }^{2}$ Laboratory of Pharmaceutical Biotechnology, Faculty of Pharmacy, Universitas Indonesia, Depok, West Java 16424, Indonesia.

${ }^{3}$ Laboratory of Pharmacognosy-Phytochemisty, Faculty of Pharmacy, Universitas Indonesia, Depok, West Java 16424, Indonesia. ${ }^{4}$ Laboratory of Chemistry, Faculty of Pharmacy, Universitas Surabaya, Surabaya, East Java 60284, Indonesia. Email: arry.yanuar@ui.ac.id

Received: 29 June 2018, Revised and Accepted: 03 December 2018

\section{ABSTRACT}

Objective: The importance of SIRT1 activator's role in antidiabetic and anti-aging therapies is widely demonstrated. Drug discovery and development are time consuming. Drug design can be performed in silico using molecular dynamic approaches to accelerate and facilitate identification of the best compound candidates and their physicochemical characteristics and hit-to-lead selection.

Methods: In silico study of SIRT1 activator for complexes using of Protein Data Bank (PDB) IDs 4ZZI, 4ZZJ 4ZZH, and 5BTR and 4TO ligand. Ligandreceptor interactions and bond energies were determined using molecular docking with the AutoDock4Zn program. Then, the complex with the best bond energy was identified using a simulation of the molecular dynamics ( $50 \mathrm{~ns}$ ) using the Amber program, and values for root mean square deviation, root mean square fluctuation, and bond energy were determined using the Molecular Mechanic-Poisson Boltzmann (Generalized Born) surface area (MM-PB[GB]SA) calculation.

Results: Interaction analysis between activator ligand (4TO) and the SIRT1 receptor (PDB IDs 4ZZJ and 5BTR) revealed the ligand's selectivity for hydrophobic interaction at Leu206, Ile223, Ile227, and hydrophilic interaction at Asn226, Glu230. Hydrogen bond interactions between Glu230 and Arg234 (allosteric region) with Arg446, Val459, His473, and Asp475 (catalytic region) brought them close to the bounding substrate area. Bond energy values obtained using the MM-GB(PB)SA calculation showed 4TO interaction with 4ZZJ (MMGBSA $\Delta \mathrm{G},-31.4729--26.6756$; MMPBSA $\Delta \mathrm{G}$, -32.6292--28.486]. The bond energy value of the 4 TO interaction with 5BTR showed MMGBSA $\Delta \mathrm{G}=-40.6255--30.0653$ and MMPBSA $\Delta \mathrm{G}=-34.6713--25.9951$.

Conclusions: These findings provide important information on the target interaction of the bonds to the more selective SIRT1 activator useful for drug discovery and development.

Keywords: SIRT1 activator, Molecular docking, Molecular dynamic, Molecular mechanic/Poisson Boltzmann (generalized born) surface area.

(c) 2019 The Authors. Published by Innovare Academic Sciences Pvt Ltd. This is an open access article under the CC BY license (http://creativecommons. org/licenses/by/4. 0/) DOI: http://dx.doi.org/10.22159/ijap.2019.v11s1.19266

\section{INTRODUCTION}

SIRT1 enzyme activity has two primary roles as a target for therapy, namely SIRT1 inhibition in the catalytic region which is used in cancer treatment and SIRT1 activation in the allosteric region which is used in metabolic damage treatment, including diabetes [1,2] and anti-aging [3-5]. SIRT1 is a nicotinamide adenine dinucleotide (NAD+)-dependent deacetylase which catalyzes of deacetylating protein in lysine residues [6,7].

The structure of human sirtuin protein includes the SIRT1 isoform (UniProt accession code Q96EB6, available online: NCBI, Research Collaboratory for Structural Bioinformatics). The SIRT1 sequence comprises 747 amino acids; amino acids 1-180 are located at the $\mathrm{N}$-terminal region, amino acids $195-240$ in the allosteric region (active regulator of SIRT1), amino acids 244-512 in the catalytic region, and amino acids 513-747 in the C-terminal region [8].

Nucleotide bonds including NAD+ are found in the amino acid regions 261-280, 345-348, 440-442, and 465-467; metal bonds are present at amino acids $371,374,395$, and 398; the deacetylase area is at amino acids 244-498; and the activation area including sirtuin-activating compounds (STACs) are at amino acids 195-243 and Glu(E)230 [9,10]. Homologous sequences identical to SIRT1 and with allosteric regions obtained from the protein data bank (PDB) [6] include PDB IDs 4ZZI, 4ZZJ, 4ZZH [11], and 5BTR [12].

The structure of SIRT1 PBD ID 4ZZI comprises a ligand activator 4TQ ([3S]1,3-dimethyl-N-[3-[1,3-oxazol-5-yl]phenyl]-6-[3-[trifluoromethyl]phenyl]2,3-dihydropyrido[2,3-b]pyrazine-4[1H]-carboxamide) that interacts at the allosteric region, and ligand inhibitor 1NS 4-(4-[2-[[methylsulfonyl] amino]ethyl] piperidin-1-yl)thieno(3,2-d)pyrimidine-6-carboxamide in the catalytic region. 4TQ activators demonstrate hydrophobic interactions (HIs) at amino acids Thr219, Leu215, Ile223, and hydrogen bond at Asn226. The 1NS inhibitor shows HIs at residues Phe297 and His363 and hydrogen bonds at Phe273, Ile347, and Asp348.

The structure of SIRT1 PDB ID 4ZZJ (www.rcsb.org/structure/4ZZJ) shows interactions between $4 \mathrm{TQ}$ at the allosteric region of the HI of amino acids Thr219 and Ile223, hydrogen bonding at Asn226, and with CNA (carba-nicotinamide-adenine-dinucleotide) ligand at the catalytic region.

The active conformation model of SIRT 1 comprises a STAC complex with the Ac-p53 substrate, indicating the presence of amino acid hydrogen bond interactions at Glu230 and Arg446. The structure of the complex of SIRT1 with STAC compounds shows binding of STAC-1 to the STAC binding domain (SBD). Binding of STAC encourages SBD interaction with the central catalytic region to the substrate, increasing the activity of SIRT1 and stabilizing the deacetylase activity. Replacement of Glu (E) 230 with Lys residue disrupts enzymatic activation by reducing the electrostatic interactions between Glu230 in the SBD and Arg446 at the substrate-binding site [13].

The structure of SIRT1 PDB ID 4ZZH (www. rcsb. org/structure/4ZZH) shows HIs of STACs (4TO) ([4S]- $N$-[3-[1,3-oxazol-5-yl]phenyl]-7-[3[trifluoromethyl] phenyl]-3,4-dihydro-1,4-methanopyrido[2,3-b][1,4] diazepine-5[2H]-carboxamide) with Leu 215, Thr219, and ILE223 and hydrogen interactions with Asn226 in the allosteric region. 
The structure of SIRT1 PDB ID 5BTR (www.rcsb.org/structure/5btr) is the SIRT1 activator located in the allosteric region, a complex containing three resveratrol ligands (STL), and shows hydrogen bonding interactions at Gln222, Asn226, lys444, Asp298, Asp292, and Glu230 and HIs at Gly415, Ile223, Gln294, Ile223, and Arg446.

Interaction of 7-amino-4-methyl coumarin with 5BTR shows HIs at Phe412, Val445, His363, and Phe414 and hydrogen bond interactions at Arg446, Asn226, Gly415, Asp150, Glu416, and Val412 [12].

Therefore, the present study used an in silico study with molecular docking and dynamic simulations of the SIRT1 enzyme (PDB IDs 4ZZI, 4ZZJ, 4ZZH, and 5BTR) to determine the bonding interactions of SIRT1 required for ligand selectivity and selective identification of bond interactions and target bond interactions with biological function $[14,15]$.

\section{MATERIALS AND METHODS}

\section{Materials}

The following materials were used: IntelXeon $(\mathrm{R})$ central processing unit E5620 at $2.40 \mathrm{GHz} \times 16$, processor $2.6 \mathrm{GHz}$ Intel Core i7, random access memory $32 \mathrm{~GB} 1600 \mathrm{MHz}$ DDR3, and Graphics Intel Iris 1536 MB. The operating system used was Linux Ubuntu 12.04 LTS with an uninterrupted power supply.

\section{Protein preparation}

Preparation of macromolecular three-dimensional structure was done by downloading macromolecules SIRT1 (PDB IDs: 4ZZI, 4ZZJ, 4ZZH, and 5BTR) was obtained from the Research Collaboratory for Structural Bioinformatics PDB (http://www. rscb. org/pdb) [16]. All macromolecules were clean-up from ligands, substrates, ions, and waters before minimization with Amber program [17].

\section{Ligand preparation}

The ligands used were the 4T0 crystals of macromolecular SIRT1 (PDB IDs: 4ZZH). Minimization of ligand used the AMBER program, and 1NS compound (SIRT1 inhibitor) was used as a negative control.

\section{Molecular docking validation}

Autodock4Zn was used to perform molecular docking in this study $[18,19]$. Validation of the autodock4Zn program measured the root mean square deviation (RMSD) value analyzed using the visual molecular dynamics (VMD) program and the initial cocrystal structure of PDB against the conformational crystal positioning after molecular redocking was done.

\section{Molecular docking}

Molecular docking was performed using AutoDock4Zn, with ligandmacromolecular grid binding sites by determining npts and gridcenter.

Analysis of the docking results in the form of affinity and RMSD binding values (for docking performance validation) and visualization of docking results to analyze ligand interaction with macromolecules used LigandScout (Inte:Ligand, Austria) [20] and CHIMERA (UCSF, USA), respectively [21].

\section{Molecular dynamics}

The best compounds for molecular docking were selected for the molecular dynamics study, and the simulation was performed using Amber12 [17] for 50 ns.

General amber force field (GAFF) was used for ligand preparation [13]. Ligands and macromolecules topologies and coordinates were created in a vacuum and explicit waters environment. At this stage, the ligand structure was provided with an AM1-bond charge correction charge [14] using Antechamber software (UCSF, USA) accessed via PuTTY. The file output was obtained in the form of *.mol2, and the antechamber result created a *frcmod file.

Preparation of parameters and coordinates of macromolecule files containing Zn was performed online (http://mayoresearch. mayo.edu/ mayo/research/camdl/zinc_protein.cfm).
Preparation of peptide minimization (substrate) used parameters and coordinates of peptide-containing (NArg His Lys Lys Leu Met CPhe) macromolecular files [22]. Preparation for the formation of $\mathrm{NAD}^{+}$used $\mathrm{NAD}^{+}$as a cofactor with a positive charge. The creation of macromolecules with $\mathrm{NAD}^{+}$required $\mathrm{NAD}^{+}$.lib, and $\mathrm{NAD}^{+}$.frcmod parameter files were obtained from Ross Walker, and the coordinates were altered using the coordinates of the $\mathrm{NAD}^{+}$file associated with the macromolecules. Further complexes of ligand: $\mathrm{Zn}$ : NAD': Macromolecules were used as topological and coordinate files with the addition of water molecules TIP3PBOX $12 \AA^{3}$, followed by minimization, heating, density, equilibration, and production.

Before continuing the dynamics simulation, verification of the system was balanced using the command "process_mdout. pl" to extract useful information from the output file: heat. out, density. out, and equil. out. A balanced system was seen from the temperature, density, and RMSD.

After the balanced system was observed, production was continued for 50 ns. The ptraj program from AmberTools and VMD was used to perform trajectory analysis of the molecular dynamic simulation results [15]. The parameters analyzed were RMSD and root mean square fluctuation (RMSF), and the hydrogen bond conditions were analyzed using VMD program. Hydrogen bonds with an occupancy of $>50 \%$ of the overall data from the hydrogen bond analysis were selected. The distance between the donor and acceptor of the hydrogen bond was set at $3.0 \AA$, and the cutoff angle was set at $60^{\circ}$. The Molecular Mechanic-Poisson Boltzmann (Generalized Born) surface area (MM$\mathrm{PB}[\mathrm{GB}] \mathrm{SA})$ method was used to calculate the binding energy [23].

\section{RESULTS AND DISCUSSION}

The present study was performed by visualizing 4TO cocrystal interactions with SIRT1 macromolecules (SIRT1 PDB IDs 4ZZI, 4ZZJ, and 4ZZH) and one with three STLs (PDB ID 5BTR). Analysis of complex compound interactions (STACs) with SIRT1 activator receptors in the allosteric region was used to examine the bonding interactions that play an important role in ligand selectivity. Activator compounds act as sirtuin activators bound to the amino acid allosteric site at residues 183-243 and generally interact at the hydrophobic chains at Thr209, Pro211, Pro212, Leu215, Thr219, Ile223, and Ile227 at the shape of the (helix-turn-helix) amino acid and one hydrophilic interaction at Asn226 (Supplemental Fig. 1).

Visualization of macromolecular interactions with SIRT1 PDB IDs 4ZZI, 4ZZJ, 4ZZH, and 5BTR showed differences in the position of the allosteric region receptors. Residues at Glu230-Asn241 showed a flexible helix shape. As Fig. 1 shows, the superimposition of SIRT1 PDB ID 4ZZI demonstrates a more closed form between the allosteric and catalytic regions, containing 4TO ligand crystals in the allosteric region and 1NS ligand crystals in the catalytic region. SIRT1 PDB ID 4ZZJ contains CNA and 4TO ligand crystals in the allosteric region, and the allosteric area form was catalytically more open than that of 4ZZI. PDB $4 \mathrm{ZZH}$ showed an allosteric and catalytic form that was more open and contained only 4TO ligand crystals in the allosteric region. PDB 5BTR contained resveratrol compounds in the allosteric region adjacent to the catalytic region.

\section{Validation of molecular docking}

In the present study, AutoDock4Zn was used to visualize molecular docking. Validation of this Autodock program measured the RMSD value analyzed using the VMD program. The initial cocrystal PDB structure was analyzed against the conformational crystal positioning after molecular docking restarted. The RMSD values of $4 \mathrm{TO}$ as a crystal were $0.81 \AA, 0.79 \AA$, and $0.89 \AA$ for $4 \mathrm{ZZI}, 4 \mathrm{ZZJ}$, and $4 \mathrm{ZZH}$, respectively.

\section{Molecular docking of macromolecular 4ZZI}

The macromolecule receptor of 4ZZI has ligand crystals in the catalytic region as an activator. This ligand was 4TO ([4S]- $N$-[3-[1,3oxazole-5-yl] phenyl]-7-[3-[trifluoromethyl] phenyl]-3,4-dihydro-1,4methanopyrido $[2,3-\mathrm{b}][1,4]$ diazepine-5 $[2 \mathrm{H}]$-carboxamide) in the 
allosteric region as an activator. The molecular docking results of the $4 \mathrm{TO}$ obtained bond energy values of $\Delta \mathrm{G}=-6.86 \mathrm{kcal} / \mathrm{mol}$ and a $\mathrm{Ki}$ value of $9.36 \mu \mathrm{M}$ (predicted binding interaction).

Fig. 2 shows the 4 TO ligand crystal compound interaction. 4 TO bonded to the activating region of essential amino acids Ile223 and Ile227. The complex showed four hydrophobic features at amino acids namely Leu206 (HI), Thr219 (HI), Ile223 (HI), Ile227 (HI), Asn226 (hydrogen bond acceptor [HBA]), and Glu230. Docking of the 1NS compound (SIRT1 inhibitor) as a negative control on the allosteric region showed interactions at Thr219 (HBD) and Ile227 (HBD) and no HIs (Supplemental Fig. 2)

\section{Molecular docking of macromolecular 4ZZJ}

The AutodockZn program was used to analyze docking of the ligand crystal molecule and the active compound SIRT1 with 4ZZJ. The results obtained from the process showed a bond energy value of $\Delta \mathrm{G}=-6.94 \mathrm{kcal} / \mathrm{mol}$, a $\mathrm{Ki}$ value of $8.24 \mu \mathrm{M}$ (predicted binding interaction), and the complex interaction of ligand and receptor bonds.

Fig. 3 shows 4 TO compound interaction. The complexes demonstrate four hydrophobic features at amino acids namely Leu206 (HI), Thr209,
Leu215 (HI), Thr219 (HI), Ile223 (HI), and Ile227 (HI) and show acceptor hydrogen bonding interactions (HBA) at Asn226. Docking of the 1NS compound (SIRT1 inhibitor) as a negative control on the allosteric region showed interactions at Gln222 (HBD) and Thr219 (HBD) and no HIs (Supplemental Fig. 2).

Molecular docking of macromolecular 4ZZH

The AutodockZn program was used to generate molecular docking of the crystal ligand molecule and the active compound SIRT1 with $4 \mathrm{ZZH}$. The results were obtained using a bond energy value of $\Delta \mathrm{G}=-7.61 \mathrm{kcal} / \mathrm{mol}$ and $\mathrm{Ki}=5.6 \mu \mathrm{M}$ (predicted binding interactions)

The 4TO compound showed interactions with four hydrophobic features of the amino acids namely Leu206 (HI), Leu215, Thr219 (HI), Ile223 (HI), and Ile227 (HI) and showed acceptor hydrogen bonding interactions (HBA) at Thr209 (HI) residues.

4TO compound interaction with macromolecules is shown in Fig. 4, showing interaction with four hydrophobic features at amino acids namely Leu206 (HI), Thr209, Leu215 (HI), Thr219 (HI), Ile223 (HI), and Ile227 (HI)

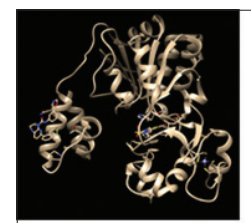

4ZZI

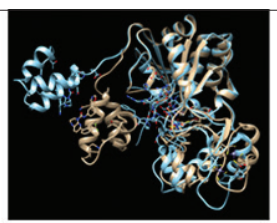

4ZZI-4ZZJ

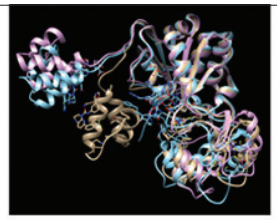

4ZZI-4ZZJ4ZZH

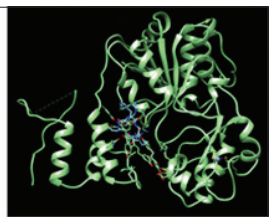

5BTR

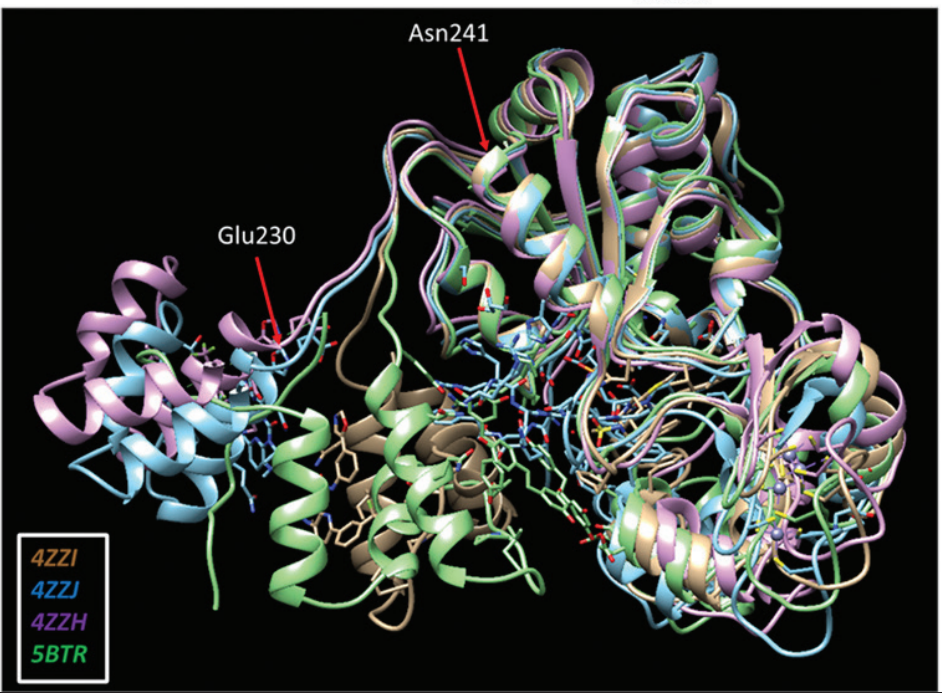

Fig. 1: Visualization superimposition crystal structure of protein data bank (4ZZI, 4ZZJ, 4ZZH, and 5BTR) shows the flexibility of allosteric region

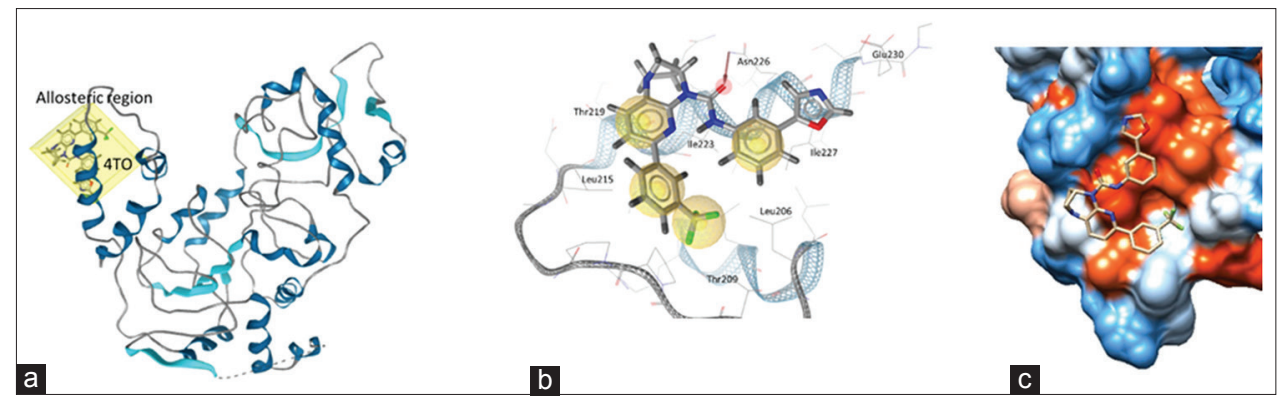

Fig. 2: (a) Molecular docking of 4T0:4ZZI at the allosteric region. (b) Visualization of 4TO:4ZZI complex interactions at Thr219, Ile223, Ile227, Asn226 (hydrogen bond acceptor), and Glu230 using the LigandScout program. (c) Visualization of the 4TO:4ZZI complex using a mesh surface image generated by CHIMERA (hydrophilic, blue; hydrophobic, orange) 
Molecular docking of macromolecular 5BTR

Molecular docking 4TO with 5BTR using the AutodockZn program was obtained using a bond energy value of $\Delta \mathrm{G}=-13.1 \mathrm{kcal} / \mathrm{mol}$ and $\mathrm{Ki}=2.51 \times 10^{-4} \mu \mathrm{M}$ (predicted binding interaction).

The 4TO ligand revealed bonds at the activating regions of essential amino acids Ile223 and Ile227. The complex showed hydrophobic features at amino acids namely Leu206 (HI), Thr219 (HI), Ile223 (HI), and Ile227 (HI) (Fig. 5). Docking of the 1NS compound (SIRT1 inhibitor) as a negative control on the allosteric region showed interactions at Leu206 (HBD) and no HIs (Supplemental Fig. 2).

Molecular dynamic simulation

Molecular dynamic simulation of 4TO compounds against 4ZZI, 4ZZJ, 4ZZH, and 5BTR receptors was performed for 50 ns using the Amber program. Analysis of the dynamics simulation result was carried out considering RMSD, RMSF, and hydrogen bonding conditions, and the binding energy was calculated using the MMGB/PBSA method.

\section{RMSD}

Conformational changes of the 4TO compound during the simulation were seen from the RMSD values. The RMSD curve for 50 ns showed a change in the stability of the 4 TO complex dynamic simulation that corresponded to 4ZZI, 4ZZJ, 4ZZH, and 5BTR receptors. In Fig. 6, the 4T0:4ZZI, 4T0:4ZZJ, and 4T0:5BTR complexes showed an RMSD range of $2 \AA$, whereas the $4 \mathrm{TO}: 4 \mathrm{ZZH}$ complex had a RMSD range of $3 \AA$.

\section{RMSF}

RMSF is the measure of the deviation between the atomic positions of each protein residue, i.e., the difference in fluctuations in the movement of each residue during the simulation is measured for 50 ns. Fig. 7 shows the RMSF value of the complex molecular dynamic simulation between the 4TO ligand (ligand crystal) and 4ZZI, 4ZZJ, 4ZZH, and 5BTR receptors. The $4 \mathrm{ZZH}$ receptor showed fluctuations of movement in the $\mathrm{N}$-terminal domain of all RMSF compared with 4ZZI, 4ZZJ, and 5BTR receptors that did not show fluctuations, indicating that binding of the 4TO compound to the receptor is more stable.

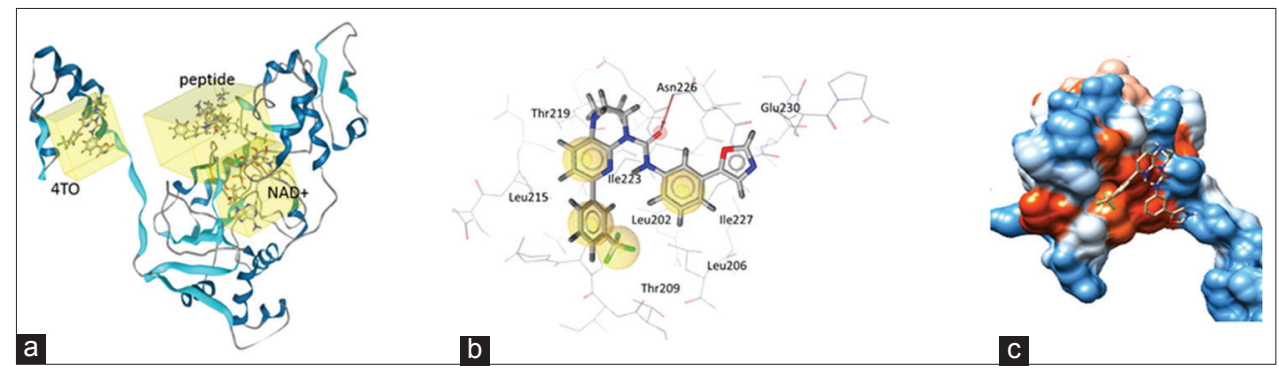

Fig. 3: (a) Molecular docking of 4TO to 4ZZJ at the allosteric region. (b) Visualization of 4TO:4ZZJ complex interactions at Leu206

(hydrophobic interaction), Thr209, Leu215 (hydrophobic interaction), Thr219 (hydrophobic interaction), Ile223 (hydrophobic interaction), Ile227 (hydrophobic interaction), and Asn226 (hydrogen bond acceptor) using the LigandScout program. (c) Visualization of the 4T0:4ZZJ complex using a mesh surface image generated by CHIMERA (hydrophilic, blue; hydrophobic, orange)

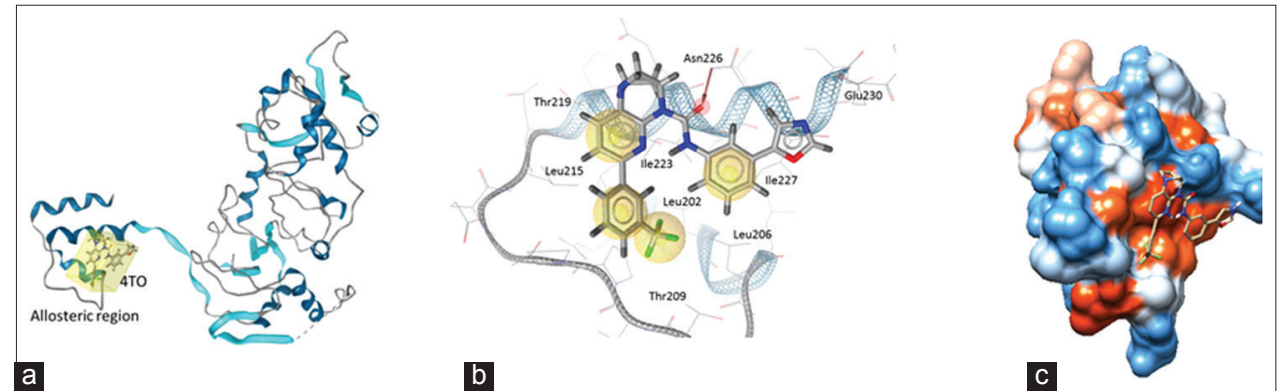

Fig. 4: (a) Molecular docking of 4TO compound with protein data bank 4ZZH at the allosteric region. (b) Visualization of 4T0:4ZZH complex interactions at amino acids Leu206 (hydrophobic interaction), Thr209, Leu215 (hydrophobic interaction), Thr219 (hydrophobic interaction), Ile223 (hydrophobic interaction), and Ile227 (hydrophobic interaction) using the LigandScout program. (c) Visualization of 4T0:4ZZH complex using a mesh surface image generated by CHIMERA (hydrophilic, blue; hydrophobic, orange)
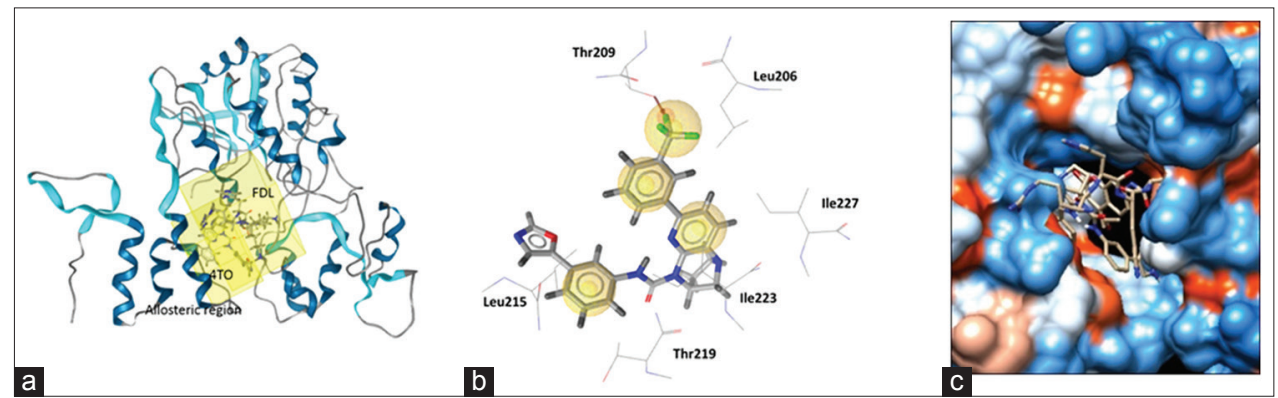

Fig. 5: (a) Molecular docking of compound 4T0:5BTR at the allosteric region. (b) Visualization of 4T0:5BTR complex interactions at Leu206, Thr209 (hydrogen bond acceptor), Leu215 (hydrophobic interaction), Thr219 (hydrophobic interaction), Ile223 (hydrophobic interaction), and Ile227 (hydrophobic interaction) using the LigandScout program. (c) Visualization of 4T0-5BTR complex using a mesh surface image generated by CHIMERA (hydrophilic, blue; hydrophobic, orange) 


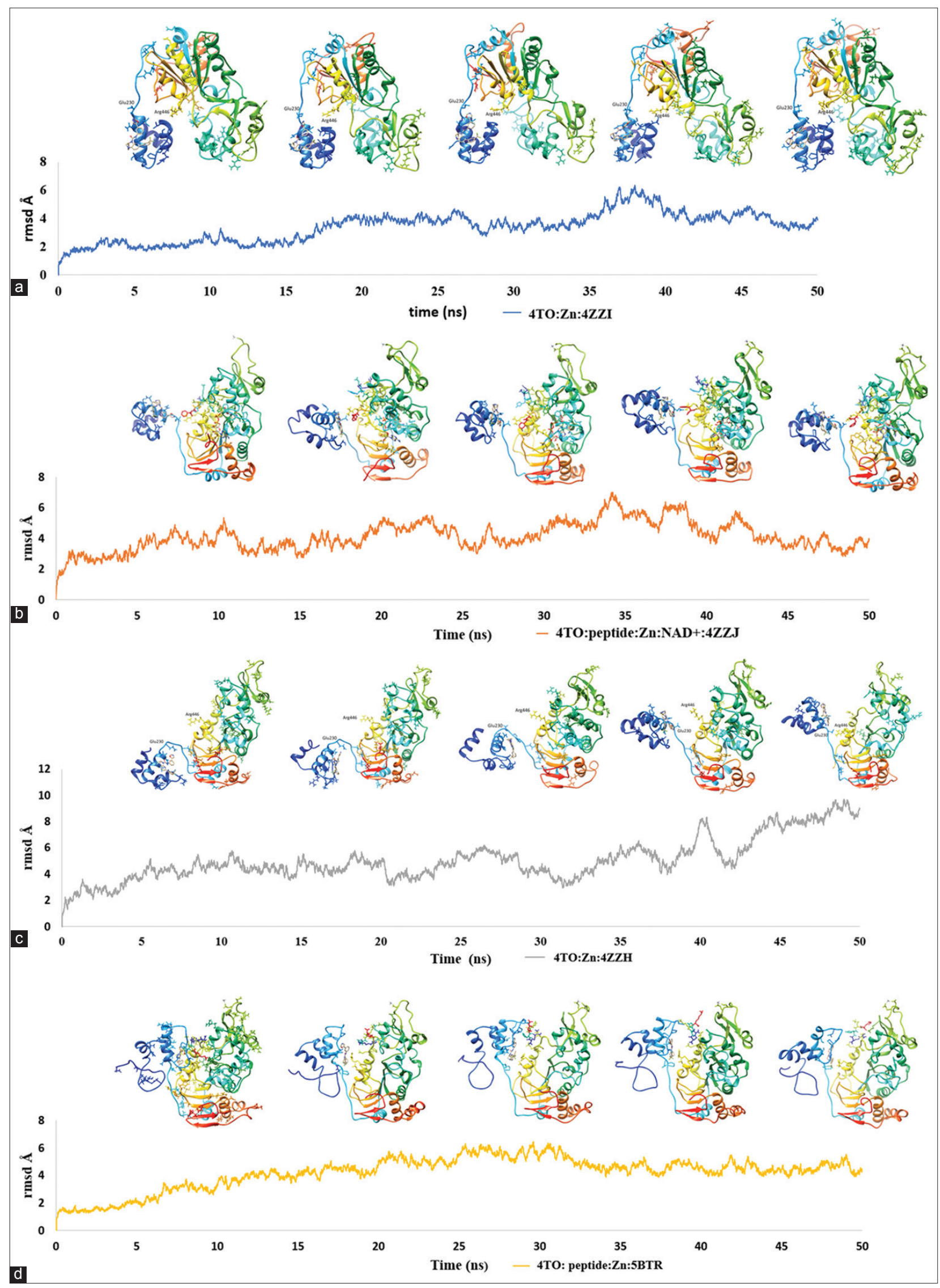

Fig. 6: Root mean square deviation and conformational changes of receptor structure at an interval of $10 \mathrm{~ns}$ (a). Zn:4ZZI, (b). peptide: Zn: NAD+:4ZZJ, (c). Zn:4ZZH, and (d). peptide: Zn:5BTR with 4 TO at $300 \mathrm{~K}$ 


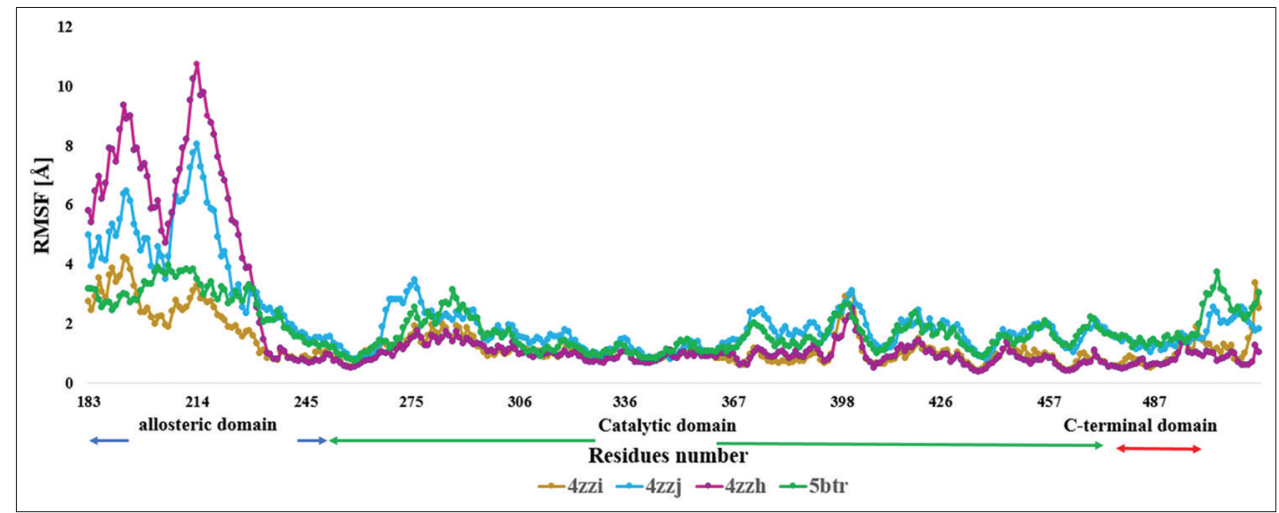

Fig. 7: Root mean square fluctuation complex of 4TO ligand with 4ZZI, 4ZZJ, 4ZZH, and 5BTR macromolecules

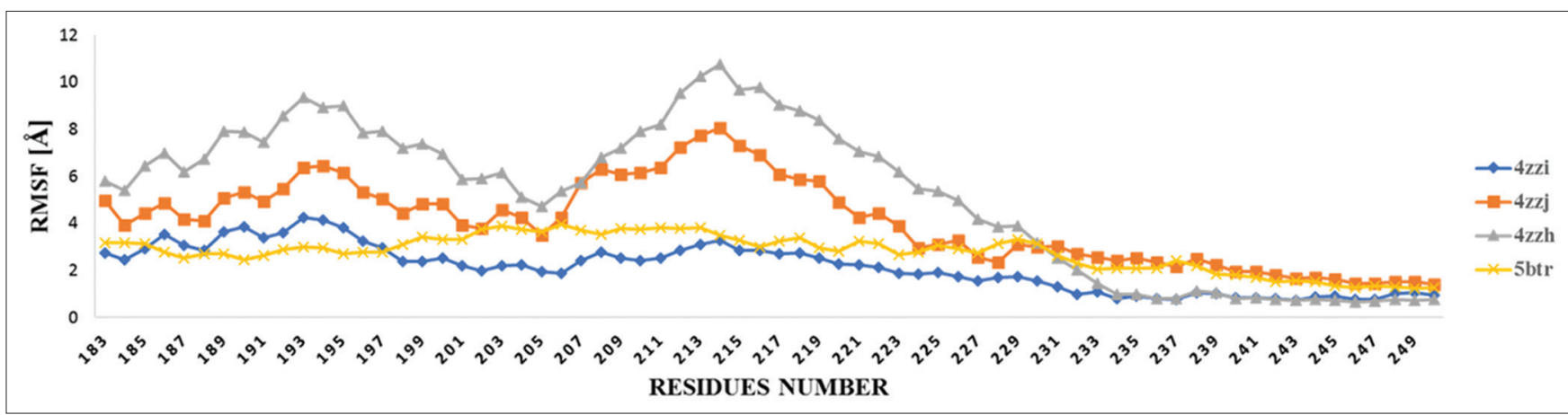

Fig. 8: Root mean square fluctuation of the backbone atoms from 4ZZI, 4ZZJ, 4ZZH, and 5BTR complex with 4 TO in the allosteric domain

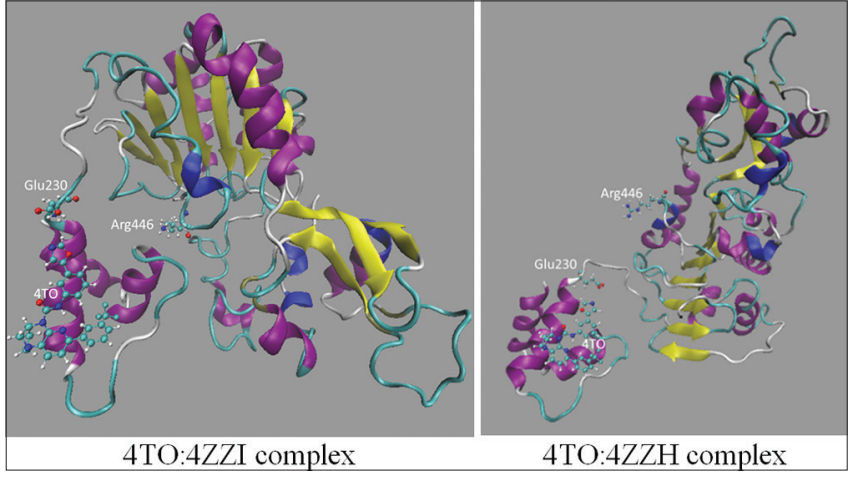

Fig. 9: Snapshot dynamic simulation of 4TO interaction with 4ZZI and $4 \mathrm{ZZH}$ receptors, whereas Glu230 residue (allosteric region) with Arg446 (catalytic region) did not show hydrogen bonding

Fig. 8 shows the RMSF of the backbone atom in a dynamic simulation system between $4 \mathrm{TO}$ ligands with 4ZZI, 4ZZJ, 4ZZH, and 5BTR receptors for $50 \mathrm{~ns}$ in allosteric regions. The 4TO ligand bond with the 4ZZI receptor and 5BTR on Leu206, Leu215, Thr219, Ile223, Asn226, Ile227, and Glu230 residues did not show fluctuations (low RMSF values), indicating that binding occurred in the residue. These data correspond to the bonding interaction in ligand crystals 4ZZI [7] and 5BTR [8]. The bond interaction between the 4TO ligand and the 4ZZJ receptor shows a low RMSF value at Leu206, Ile223, Asn226 Ile227, and Glu230, demonstrating strong binding to the residue. These data correspond to the bonding interaction of the 4ZZJ ligand crystal [7]. The binding interaction between the 4TO ligand and the 4ZZH receptor shows high RMSF values at Ile223, Asn226, Ile227, and Glu230, showing no strong binding of such residues, as in the crystal ligands of 4ZZH [7].

\section{Hydrogen bond analysis}

As an activator of SIRT1 PDB ID 4ZZJ, the 4TO ligand crystal shows the presence of hydrogen bond interactions at Arg234-Asp475 (NH-O)

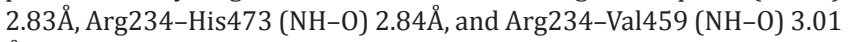
$\AA$ [7]. The VMD program was used to perform hydrogen bond strength analysis (\% occupancy) of 4TO with SIRT1 PDB IDs 4ZZI, 4ZZJ, 4ZZH, and 5BTR from molecular dynamic simulations for 50 ns. Based on the molecular dynamic simulations, 4TO in complex with 4ZZ1 and 4ZZH receptors did not show any hydrogen bond interactions between Glu230 (allosteric regions) and Arg446 (catalytic regions).

Fig. 9 shows the binding of $4 \mathrm{TO}$ ligand to the allosteric region but not to the activator of SIRT1.

The results of the hydrogen bond strength (\% occupancy) in the complex of the 4TO ligand with 4ZZJ receptor indicated the presence of important amino acid interactions, namely Glu230-Arg446, 51\%; Arg234-Asp475, 65.1\%; Arg234-His473, 22.50\%; and Arg234-Val459, 11.7\%. The hydrogen bond strength (\% occupancy) in the complex of the 4TO ligand with the 5BTR receptor showed the presence of important amino acid interactions, namely Glu(E)230-Arg(R)446, 65.80\%; $\operatorname{Arg}(\mathrm{R}) 234-$ Asp(D)475, 70.9\%; Arg(R)234-His(H)473, 33.3\%; and Arg234-Val459, $4.9 \%$ (Fig. 10). The results of this analysis indicate that the SIRT1 activator is showed by hydrogen bond interactions between Glu230 and Arg234 (allosteric region) with Arg446, Val459, His473, and Asp475 (catalytic region) which are close to the bound substrate region.

\section{Free energy calculation $(\Delta G)$}

The result of the $\Delta G$ bond energy calculated from the molecular docking with AutoDock4Zn was then recalculated to determine the free bonding energy from the 4TO compound with SIRT1 PDB IDs 4ZZI, 4ZZJ, 4ZZH, and 5BTR using the MM-GB(PB)SA method, with simulation dynamics carried out for $50 \mathrm{~ns}$. This was performed to ensure that the bond energy was more selective [23], and the energy value of the 4TO ligand bond with 4ZZJ receptor using the MMGBSA method was calculated, while MMPBSA measured bond energy on simulation dynamics for $50 \mathrm{~ns}$. 


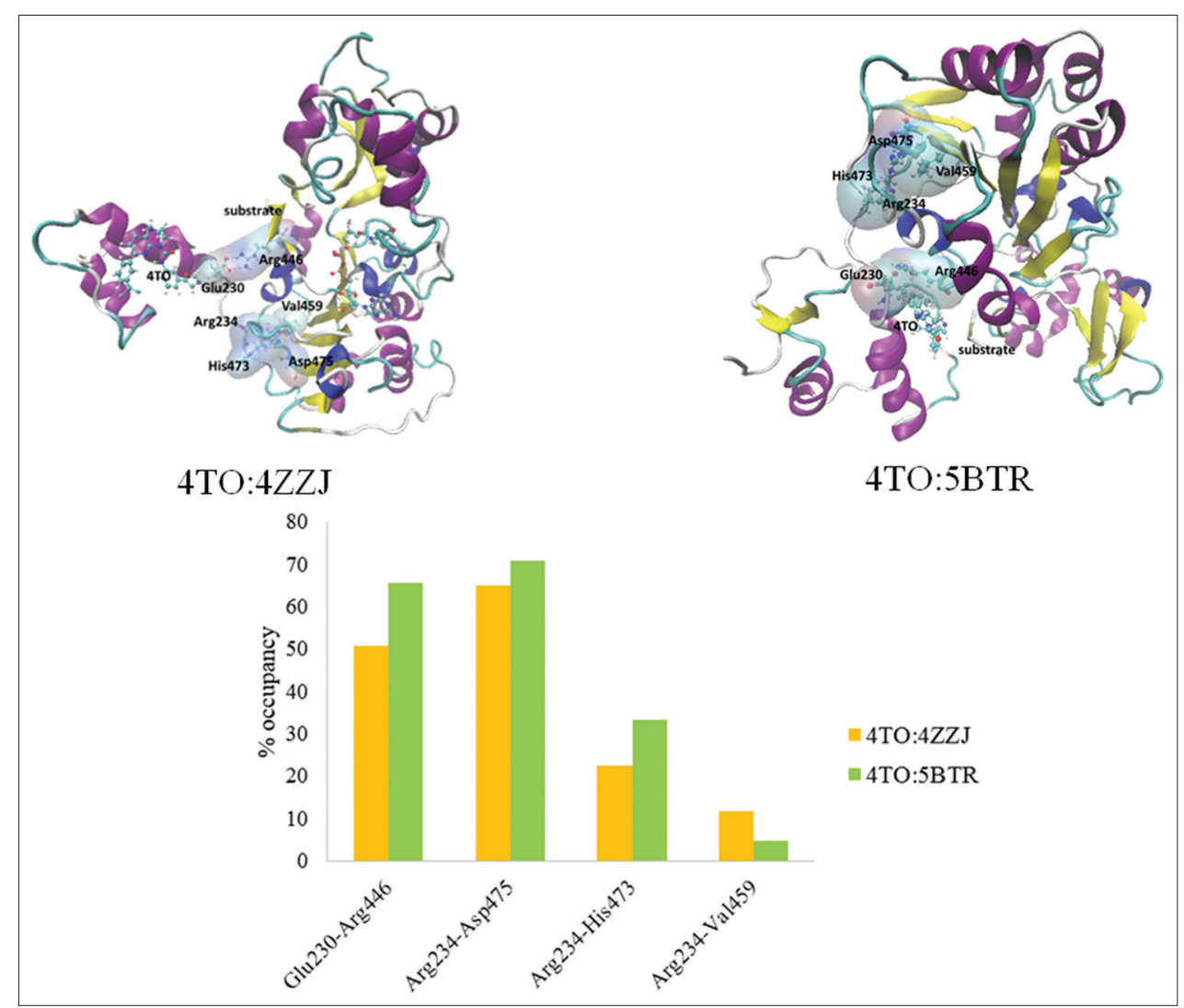

Fig. 10: Hydrogen bond strength (\% occupancy) of important amino acid interactions in the complex of 4TO:4ZZJ and 4TO:5BTR
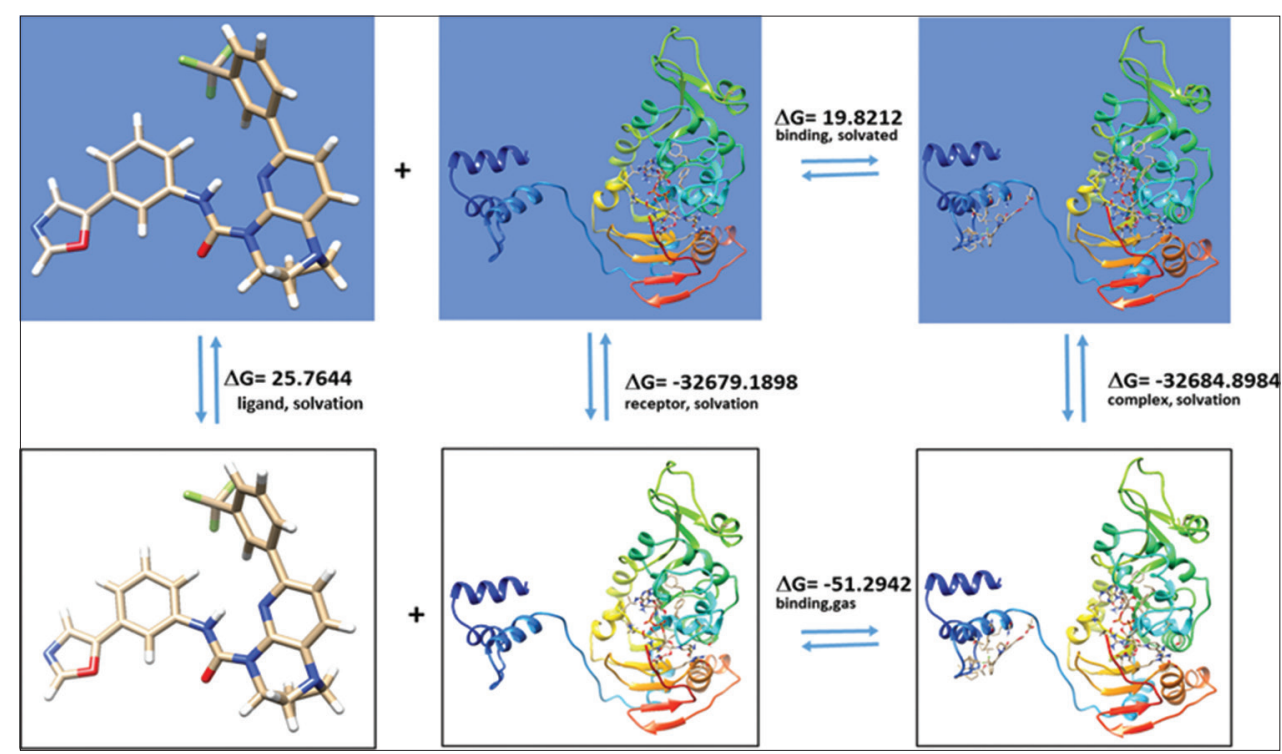
receptor, solvation $\Delta \mathrm{G}=-32684.8984$
complex, solvation
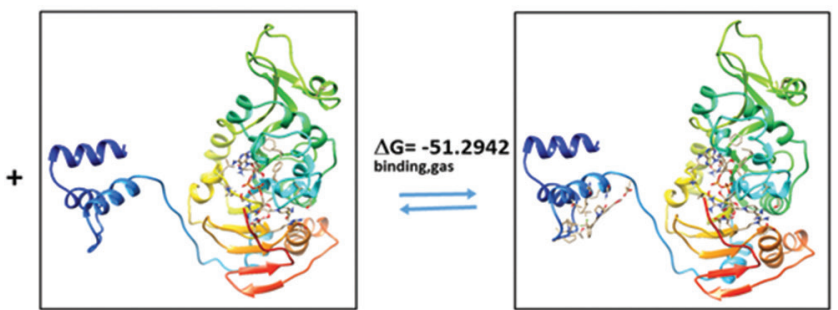

Fig. 11: Calculation of free bonding energy (MMGBSA) in 4T0: peptide:Zn: NAD+:4ZZJ complex. The calculation of the compound complex of $4 \mathrm{TO}$ ligand crystal with peptide: $\mathrm{Zn}: \mathrm{NAD}+: 4 \mathrm{ZZJ}$ found a value of MMGBSA ( $\Delta \mathrm{Gtotal})=-31.4729 \mathrm{kcal} / \mathrm{mol}, \Delta \mathrm{Gtotal}=\Delta \mathrm{Ggas}+\Delta \mathrm{Gsolv}=$ $-51.2942+19.8212=-31.479 \mathrm{kcal} / \mathrm{mol}$

The calculation of the compound complex of 4 TO ligand crystal with peptide Zn: NAD+:4ZZJ found a value of MMGBSA $\left(\Delta \mathrm{G}_{\text {total }}\right)=-31.4729 \mathrm{kcal} / \mathrm{mol}$, $\Delta \mathrm{G}_{\mathrm{total}}=\Delta \mathrm{G}_{\mathrm{gas}}+\Delta \mathrm{G}_{\mathrm{solv}}=-51.2942+19.8212=-31.479 \mathrm{kcal} / \mathrm{mol}$. Fig. 11 shows an example of the calculation. Fig. 12 shows the overall results.

The 4T0:Zn:4ZZI bond energy was measured for $50 \mathrm{~ns}$ at a temperature of $300 \mathrm{~K}$, which showed a range of bond energy values as follows: MMGBSA $\Delta G=1.9479 \mathrm{kcal} / \mathrm{mol}(-28.4461--26.4982)$ and MMPBSA $\Delta \mathrm{G}=1.4592 \mathrm{kcal} / \mathrm{mol}(-30.0343--28.5751)$. The bond energy of
4T0:peptide:Zn: NAD+:4ZZJ showed a range of bonding energy values as follows: MMGBSA $\triangle \mathrm{G}=4.7973 \mathrm{kcal} / \mathrm{mol}(-31.4729--26.6756)$ and MMPBSA $\Delta G=4.1432 \mathrm{kcal} / \mathrm{mol}(-32.6292--28486)$. The 4TO:Zn:4ZZH bond energy indicates the range of bond energy enzymes as follows: MMGBSA $\Delta \mathrm{G}=10.9523 \mathrm{kcal} / \mathrm{mol}(-36.2616--25.3093)$ and MMPBSA $\Delta G=10.8905 \mathrm{kcal} / \mathrm{mol}(-37.4334--26.5429)$. The bond energy of 4TO:Zn:5BTR:Ac:peptide showed a range of energy values as follows: MMGBSA $\triangle G=10.56 \mathrm{kcal} / \mathrm{mol}(-40.6255-30.0653)$ and MMPBSA $\Delta \mathrm{G}=8.6762 \mathrm{kcal} / \mathrm{mol}(-34.6713--25.9951)$. The results obtained from 


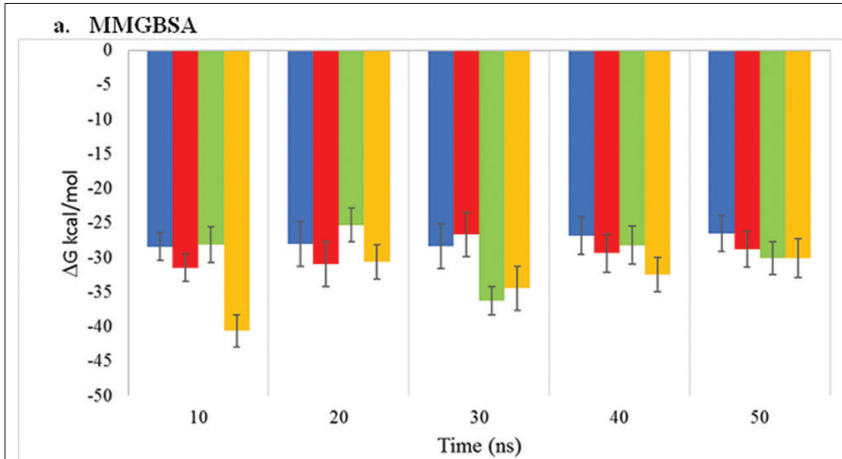

"4TO:Zn:4ZZI $=4 \mathrm{TO}:$ peptide:Zn:NAD+:4ZZJ $\approx 4 \mathrm{TO}: \mathrm{Zn}: 4 Z Z H \equiv 4 \mathrm{TO}:$ peptide:Zn:5BTR

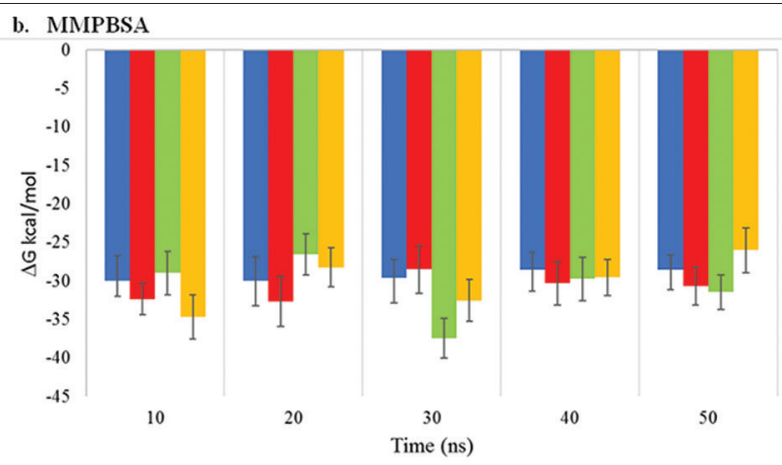

"4TO:Zn:4ZZI $=4$ TO:peptide:Zn:NAD+;4ZZJ $=4$ TO:Zn:4ZZH $=4$ TO:peptide:Zn:5BTR

Fig. 12: Results of the 4 TO bond energy with 4ZZI, 4ZZJ, 4ZZH, and 5BTR receptors using the MMGBSA and MMPBSA method for $50 \mathrm{~ns}$

the four different receptors (4ZZI, 4ZZJ, 4ZZH, and 5BTR) showed that the strength of the 4TO bond to 5BTR was stronger than that of the other receptors because the receptor form was more closed. A small difference in MM-GB(PB)SA range values was seen in the interaction between 4TO:Zn:4ZZI.

\section{CONCLUSIONS}

The present study describes an in silico study of SIRT1 bond interaction using a simulation approach of molecular dynamics over 50 ns using the Amber program. Analysis of activator ligand (4TO) binding to the SIRT1 receptor (PDB IDs 4ZZJ and 5BTR) showed selectivity of the ligand by marked hydrophobic bond features on Leu206, Ile223, Asn226, Ile227, and Glu230 of the 4ZZJ and 5BTR receptors.

Hydrogen bond interactions between Glu230 and Arg234 (allosteric regions) with Arg446, Val459, His473, and Asp475 (catalytic areas) ensured that they became close to the bounding substrate area. The bond energy values obtained for 4TO interacting with 4ZZJ using the MM-GB(PB)SA calculation using AMBER were as follows: MMGBSA $\Delta \mathrm{G}=-31.4729--26.675$ and MMPBSA $\Delta \mathrm{G}=-32.6292--28.486$. The bond energy values of the $4 \mathrm{TO}$ interaction with $5 \mathrm{BTR}$ were as follows: MMGBSA $\Delta \mathrm{G}=-40.6255--30.0653$ and MMPBSA $\Delta \mathrm{G}=-34.6713-$ -25.9951. These results are important for drug discovery and development as they give insight into target interaction of the bonds to the more selective SIRT1 activator [24-26].

\section{ACKNOWLEDGMENTS}

We thank to the Universitas Indonesia for the TADOK 2018(Tugas Akhir Hibah Doktor) research grant.

\section{CONFLICT OF INTEREST}

There are no conflicts of interest to declare.

\section{REFERENCES}

1. Aditya R, Kiran AR, Varma DS, Vemuri R, Gundamaraju R. A review on SIRtuins in diabetes. Curr Pharm Des 2017;23:2299-307.

2. Kitada M, Koya D. SIRT1 in Type 2 diabetes: Mechanisms and therapeutic potential. Diabetes Metab J 2013;37:315-25.

3. Hall JA, Dominy JE, Lee Y, Puigserver P. The sirtuin family's role in aging and age-associated pathologies. J Clin Invest 2013;123:973-9.

4. Kume S, Kitada M, Kanasaki K, Maegawa H, Koya D. Anti-aging molecule, sirt1: A novel therapeutic target for diabetic nephropathy. Arch Pharm Res 2013;36:230-6.

5. Kitada M, Ogura Y, Koya D. The protective role of sirt1 in vascular tissue: Its relationship to vascular aging and atherosclerosis. Aging (Albany NY) 2016;8:2290-307.

6. Jing H, Lin H. Sirtuins in epigenetic regulation. Chem Rev 2015;115: $2350-75$

7. Bheda $\mathrm{P}$, Jing $\mathrm{H}$, Wolberger $\mathrm{C}$, Lin $\mathrm{H}$. The substrate specificity of sirtuins. Annu Rev Biochem 2016;85:405-29.

8. Sinclair DA, Guarente L. Small-molecule allosteric activators of sirtuins. Annu Rev Pharmacol Toxicol 2014;54:363-80.

9. Hubbard BP, Gomes AP, Dai H, Li J, Case AW, Considine T, et al. Evidence for a common mechanism of SIRT1 regulation by allosteric activators. Science 2013;339:1216-9.

10. Parenti MD, Bruzzone S, Nencioni A, Del Rio A. Selectivity hot-spots of sirtuin catalytic cores. Mol Biosyst 2015;11:2263-72.

11. Dai H, Case AW, Riera TV, Considine T, Lee JE, Hamuro Y, et al. Crystallographic structure of a small molecule SIRT1 activator-enzyme complex. Nat Commun 2015;6:7645.

12. Cao D, Wang M, Qiu X, Liu D, Jiang H, Yang N, et al. Structural basis for allosteric, substrate-dependent stimulation of SIRT1 activity by resveratrol. Genes Dev 2015;29:1316-25.

13. Bonkowski MS, Sinclair DA. Slowing ageing by design: The rise of $\mathrm{NAD}^{+}$and sirtuin-activating compounds. Nat Rev Mol Cell Biol 2016;17:679-90.

14. Yanuar A, Azminah EY, Andika EY, Erlina L, Syahdi RR. In silico approach to finding new active compounds from histone deacetylase (HDAC) family. Curr Pharm Des 2016;22:3488-97.

15. Sacconnay L, Carrupt PA, Nurisso A. Human sirtuins: Structures and flexibility. J Struct Biol 2016;196:534-42.

16. Rose PW, Bi C, Bluhm WF, Christie CH, Dimitropoulos D, Dutta S, et al. The RCSB protein data bank: New resources for research and education. Nucleic Acids Res 2013;41:D475-82.

17. Case DA, Darden TA, Cheatham TE, Simmerling CL, Wang J, Duke RE, R. et al. AMBER 12; 2012. Available from: http://www. ambermd.org.

18. Santos-Martins D, Forli S, Ramos MJ, Olson AJ. AutoDock4(Zn): An improved autoDock force field for small-molecule docking to zinc metalloproteins. J Chem Inf Model 2014;54:2371-9.

19. Morris GM, Huey R, Lindstrom W, Sanner MF, Belew RK, Goodsell DS, et al. AutoDock4 and autoDockTools4: Automated docking with selective receptor flexibility. J Comput Chem 2009;30:2785-91.

20. Wolber G, Langer T. LigandScout: 3-D pharmacophores derived from protein-bound ligands and their use as virtual screening filters. J Chem Inf Model 2005;45:160-9.

21. Pettersen EF, Goddard TD, Huang CC, Couch GS, Greenblatt DM, Meng EC, et al. UCSF Chimera--a visualization system for exploratory research and analysis. J Comput Chem 2004;25:1605-12.

22. Skjaerven L, Strahm Y, Petersen K, Walker R. Course in Applied Structural Bioinformatics : Amber Peptide Tutorial. 2010. Available from: http://www.ii.uib.no/ slars/bioinfocourse/PDFs/amber peptide tut.pdf.

23. Miller BR, Mcgee TD, Swails JM, Homeyer N, Gohlke H, Roitberg AE. MMPBSA.py: An efficient program for end-state free energy calculations. Am Chem Soc 2012;8:3314-21.

24. Dai H, Sinclair DA, Ellis JL, Steegborn C. Sirtuin activators and inhibitors: Promises, achievements, and challenges. Pharmacol Ther 2018;188:140-54.

25. Aldisa O, Azminah A, Erlina L, Hayun H, Yanuar A. Virtual screening of Indonesian herbal database to find sirtuin 1 activators using the docking method. Asian J Pharm Clin Res 2017;10:158-62.

26. Sowndarya R, Doss VA. Evaluation of sirtuin 3 biomarker before and after exercise regimen in chronic unpredictable mild stress-induced depressed rats. Asian J Pharm Clin Res 2019;12:180-4. 


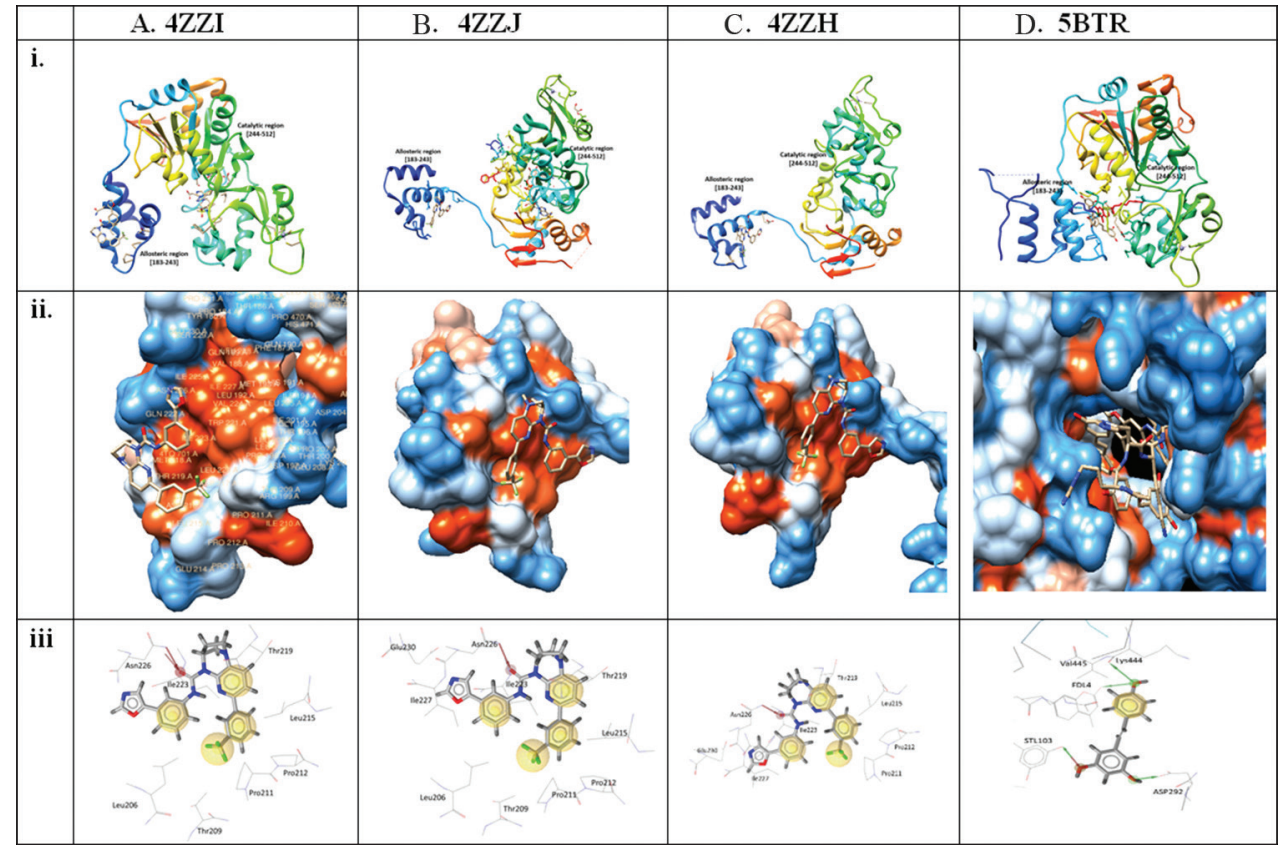

Supplemental Fig. 1: (A) (i) Visualization of 4ZZI. (ii) The structure of the 4T0:4ZZI complex shown as a mesh surface image generated by CHIMERA (hydrophilic, blue; hydrophobic, orange). (iii) Three-dimensional image generated using LigandScout. (B) (i) Visualization of 4ZZJ. (ii) The structure of the 4T0:4ZZJ complex shown as a mesh surface image generated by CHIMERA (hydrophilic, blue; hydrophobic, orange). (iii) Three-dimensional image generated using LigandScout. (C) (i) Visualization of 4ZZH. (ii) The structure of the 4TO:4ZZH complex shown as a mesh surface image generated by CHIMERA (hydrophilic, blue; hydrophobic, orange). (iii) Three-dimensional image generated using LigandScout. (D) (i) Visualization of 5BTR. (ii) The structure of the resveratrol:4ZZJ complex shown as a mesh surface image generated by CHIMERA (hydrophilic, blue; hydrophobic, orange). (iii) Hydrogen bond interaction at the allosteric region

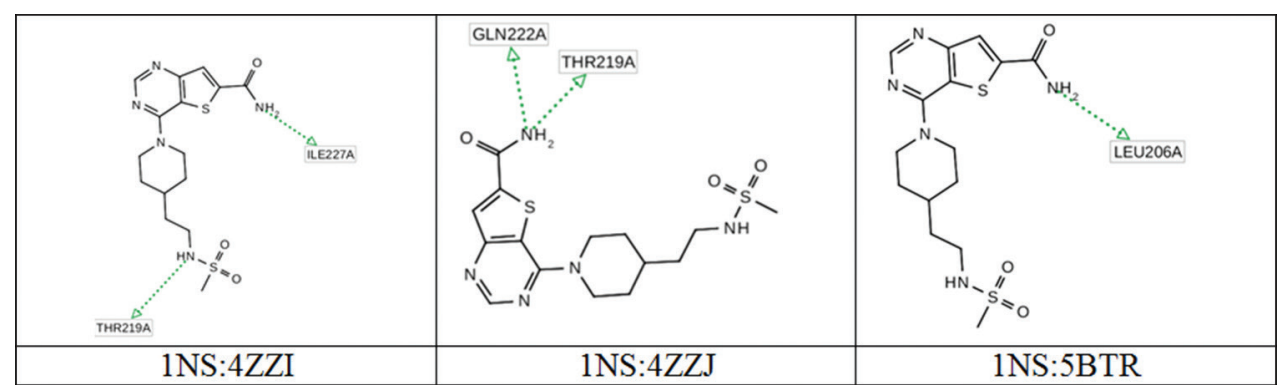

Supplemental Fig. 2: Molecular docking of the 1NS compound (SIRT1 inhibitor) as a negative control in the allosteric site 